\title{
Heart Failure with Preserved Ejection Fraction: A Review for the Clinician
}

\author{
Rabel Misbah Rameez ${ }^{1}$ and Kiyotaka Fukamachi' ${ }^{2 *}$ \\ ${ }^{1}$ Department of Internal Medicine, Cleveland Clinic, USA \\ ${ }^{2}$ Department of Biomedical Engineering, Cardiovascular Dynamics Laboratory, Lerner Research Institute, Cleveland Clinic, USA
}

Submission: July 05, 2019; Published: July 22, 2019

*Corresponding author: Kiyotaka Fukamachi, Department of Biomedical Engineering/ND20, Lerner Research Institute, Cleveland Clinic, 9500 Euclid Avenue, Cleveland, OH 44195, USA

\section{Abstract}

Almost half of patients with heart failure also have preserved ejection fraction (HFpEF) and the prevalence is increasing. $\mathrm{HFpFF}$ is diagnosed based on signs and symptoms of heart failure with ejection fraction of $\geq 50 \%$. Patients with HFpEF are a heterogeneous group with multiple associated comorbid conditions, including obesity, female gender, advanced age, and hypertension. No clear treatment guidelines exist for $\mathrm{HFpEF}$, and most therapies have not shown a clear benefit in lessening mortality. Current options include treating signs and symptoms and the associated comorbidities. Future therapeutic options have shown some promise; however, larger cohorts and more randomized controlled trials are needed.

Keywords: Heart failure; Preserved ejection fraction; Diastolic dysfunction; Myocardial stiffening.

Abbreviations: HFpEF: Heart Failure with Preserved Ejection Fraction; HFrEF: Heart Failure with Reduced Ejection Fraction; LV: Left Ventricle; LA: Left Atrium; GLP-1: Glucagon-like peptide-1; ACE: Angiotensin Converting Enzyme Inhibitors; ARB: Angiotensin Receptor Blockers; NT-ProBNP : N-terminal pro b-type natriuretic peptide; RAAS: Renin-Angiotensin-Aldosterone System; ARNi: Angiotensin Neprilysin Inhibitors

\section{Introduction}

Almost $50 \%$ of patients diagnosed with heart failure also have preserved ejection fraction [1], and the prevalence of this dual condition (HFpEF) is increasing compared with heart failure with reduced ejection fraction (HFrEF) [2]. The European Society of Cardiology (ESC) guidelines define HFpEF as the presence of heart failure symptoms in patients with an ejection fraction of $\geq 50 \%$ [3]. Diastolic dysfunction, which had been hypothesized as the cause of heart failure in these patients [4], is not universally seen, which partly explains the shift away from the prior terminology of "diastolic heart failure." Unlike its counterpart HFrEF, there are no effective treatment guidelines for HFpEF [3], leaving clinicians to treat these patients with therapies traditionally available for HFrEF, but with only limited benefit in terms of reducing morbidity and mortality rates [5]. This situation highlights our lack of understanding of HFpEF as well as the need for more effective means to manage it. In this review, we provide a systematic approach to understanding this disease with emphasis on associations, treatment, and future therapies presented in a way to help clinicians in treating their patients.

\section{Notable associations and comorbidities}

\section{Aging}

HFpEF seems to be strongly associated with old age. Like every cell in the body, cardiomyocytes also undergo aging, with gradual stiffening. As the population above age 65 has increased, so has the prevalence of HFpEF. The normal cardiac aging process includes an increase in the size of myocytes and an increase in apoptosis with a subsequent decrease in elasticity, resulting in decreased diastolic filling. An increase in collagen with thicker fibrils and more crosslinking and an increased ratio of type I to type III collagen has been seen, along with increased fibronectin and decreased elastin, contributing to cardiomyocyte stiffness [6]. A higher burden of noncardiac comorbidities (average four) is also seen in elderly patients with HFpEF compared with those with HFrEF [7].

\section{Obesity}

Obesity, especially abdominal obesity, has been associated with an increased incidence of all-cause mortality in patients 
with HFpEF. It is estimated that almost $80 \%$ of elderly patients with HFpEF are obese, and the increased prevalence of HFpEF has paralleled the obesity epidemic [7]. Excess adipose tissue challenges the heart mechanically or locally via increased pericardial restraint, low oxygen diffusion to skeletal muscle fibers, etc. Increased amounts of adipose tissue have adverse metabolic effects such as hypertension, increased insulin resistance, and dyslipidemia [7]. Last, obesity has been linked with adverse systemic effects, promoting an underlying proinflammatory state. Adipose tissue has been implicated in the production and release of proinflammatory cytokines, which are thought to be responsible for myocardial inflammation and fibrosis (thus remodeling) and for alteration in signaling pathways (leading to left ventricular [LV] dysfunction) [8].

\section{Diabetes mellitus}

Diabetes mellitus is associated with worse outcomes in patients with HFpEF as well as worsened quality of life. Diabetics with HFpEF were also found to have higher levels of N-terminal pro b-type natriuretic peptide (NT-proBNP). How much of this elevated state of NT-proBNP can be attributed to diabetes is hard to say, since patients with diabetes also have higher rates of atrial fibrillation and renal dysfunction and are more likely to be obese. Much of the elevated state seems to be part of the spectrum of the metabolic syndrome, with increased insulin resistance playing an important role in the pathophysiology [9].

\section{Chronic lung disease}

Dyspnea on exertion and decreased functional capacity are often the predominant symptoms seen in patients with HFpEF [10]. Chronic obstructive pulmonary disease is seen in almost two thirds of patients with HFpEF [11]. It is difficult to determine how much of this reduced lung capacity is due to HFpEF or to chronic obstructive pulmonary disease; both are highly prevalent diseases and share many causal factors, e.g., smoking, older age, and obesity. It is also a challenge to differentiate between the symptoms of heart failure and chronic lung disease, as they often overlap and are not specific to either disease process. In patients with cor pulmonale, diagnosis of HFpEF can become a bigger challenge. Regardless of the etiology, whether secondary to heart failure or another chronic lung disease, patients with HFpEF would benefit from lung function testing to help identify inadequately treated heart failure as well as to identify underlying lung disease amenable to specific treatment.

\section{Anemia}

Although $50 \%$ of patients with heart failure are anemic, the prevalence is higher in patients with HFpEF. Anemia, independent of other clinical factors, is associated with an increase in morbidity and mortality. It should be noted that the relationship of anemia to mortality is "J shaped"; both low and elevated hemoglobin values (>14) are associated with adverse outcomes [12]. Studies have shown a link between iron deficiency (specifically, ferritin and transferrin saturation) and decreased exercise capacity in patients with HFpEF. The Ferinject Assessment in Patients with Iron Deficiency and Chronic Heart Failure (FAIR-HF) study [13] showed that treatment with intravenous ferric carboxymaltose increased functional capacity and decreased hospitalizations, regardless of anemia and symptoms. Most patients in the study, however, had an ejection fraction of 40-45\% [13]. Treatment with epoetin alfa has not shown any benefit [14].

\section{Pathophysiology}

\section{Abnormalities in $\mathrm{LV}$ relaxation and filling}

In patients with HFpEF, relaxation as well as the elastic recoil at rest are abnormal, with inadequate enhancement during exercise. These conditions cause left atrial (LA) pressures to increase in the heart's effort to improve filling by actively pushing blood into the left ventricle. This imbalance between the ventricles also contributes to the exercise intolerance seen in HFpEF patients [15].

\section{Myocardial stiffness on a cellular level}

On a cellular level, the increased stiffness of the extracellular matrix (ECM) and of the cardiomyocytes themselves has been identified as a possible culprit for LV dysfunction during diastole. An increase in type I collagen in the ECM has been seen; this buildup is due to an increase in collagen I production and concomitant decrease in its breakdown [16], arising from a downregulation of matrix metalloproteinases and upregulation of tissue inhibitor of metalloproteinases. This phenomenon is shared by patients with LV hypertrophy secondary to hypertension as well as by those with aortic stenosis. Levels of tissue inhibitor of metalloproteinases- 1 have been studied as a marker of congestive heart failure. Increased crosslinking of type I collagen is observed as well, which also promotes stiffness in the ECM. Alterations in the phosphorylation state of titin, a large elastic cytoskeletal protein, as well as an increase in oxidative stress-induced disulfide bridges of the titin molecule are also implicated in increased cardiomyocyte stiffness [2].

\section{Endothelial inflammation and oxidative stress}

A systemic proinflammatory state associated with aging in the presence of comorbid conditions has implications on the molecular level. Endothelin adhesion molecules are upregulated as a result of coronary microvascular endothelium inflammation, which promotes reactive oxygen species (ROS) and allows for adhesion and translocation of proinflammatory cells. These ROS in turn decrease the bioavailability of nitric oxide, which in turn decreases cyclic guanosine monophosphate (cGMP) levels. This cascade leads to a decrease in protein kinase $G$ activity, which promotes hypertrophy of cardiomyocytes as well as hypo-phosphorylation of titin, contributing to increased myocardial stiffness. cGMP is also involved in facilitating crossbridge detachment by decreasing myofilament sensitivity to calcium. Abnormalities with cross-bridge detachment can lead to prolongation of the action potential, which in turn promotes cardiomyocyte stiffness and impairs relaxation. Activation of 
the angiotensin-signaling pathway has been associated with dysfunction of myocardial mitochondria and generation of ROS as well [6].

\section{Systolic function and ventricular arterial coupling}

End-systolic elastance, a measure of myocardial contractile function, is preserved in HFpEF. This fact allows for the preservation of ventricular-vascular coupling (ratio of arterial to LV elastance). This preservation also explains why arterial vasodilation improves LV systolic output in HFrEF, in which ventricular-vascular coupling is impaired, but does not show much benefit in HFpEF [15]. Due to this combined stiffening of the ventricular-vascular axis, small changes in preload or afterload are greatly amplified, which accounts for labile blood pressures [16].

Although global systolic function usually registers as normal in patients with HFpEF, some abnormalities in systolic function are seen. Sentinel studies in 2002 reported subtle impairments in systolic function observed on analysis of tissue Doppler imaging [17]. Although the impairment in systolic function is subtle, its implications are seen mostly in diastole. Reduction in long axis shortening and torsion diminish the elastic recoil characteristic of normal myocardium, thus decreasing the left ventricle's ability to pull blood into it. Consequently, LV filling becomes more dependent on the left atrium, which in turn increases pulmonary venous pressures, causing increased congestion and signs and symptoms of fluid overload. This state also dampens the left ventricle's response to exercise due to a lack of appropriate augmentation of indices of systolic performance, limiting exercise tolerance [15].

\section{Abnormalities of cardiac reserve and role of peripheral factors}

The normal response to exercise involves an increase in contractility, venous return, heart rate, and peripheral vasodilation, all of which show some abnormalities in individuals with HFpEF. Similar to abnormalities in diastolic function, diastolic reserve is also compromised in HFpEF, initiating suboptimal increases in preload volumes with exercise despite significant elevations in filling pressures [18]. Abnormalities in peripheral oxygen extraction are also seen at the level of skeletal muscle and microvasculature [19]; any success seen with exercise training is likely secondary to enhanced peripheral function [20].

\section{Diagnosis}

Diagnosis of HFpEF remains a challenge because of nonspecific signs and symptoms and the presence of a normal ejection fraction. The presence of dyspnea, decreased functional capacity, and exercise intolerance are nonspecific and can often overlap with other prevalent comorbid conditions. The ESC guidelines recommend that along with clinically suspicious signs and symptoms for HFpEF, the following should be present: normal ejection fraction on echocardiography, elevated NT-pro BNP levels, and evidence of cardiac structural or functional abnormality. In case of uncertain- ty, a stress test or an invasive measurement of elevated LV pressures may be needed for diagnostic confirmation [3].

\section{Echocardiographic assessment}

Echocardiographic evaluation is the recommended first step in the presence of clinical suspicion of HFpEF. Some form of structural or functional abnormality is often required for diagnosis: LV hypertrophy or LA dilation on echocardiography, for example. Yet almost two thirds of patients do not show any diastolic dysfunction at rest [4]. The E/e' ratio is a widely used method to evaluate diastolic dysfunction. It has its limitations, however, and its absence does not rule out HFpEF. A significant number of patients with HFpEF show normal findings at rest, yet this subpopulation might benefit from dynamic or invasive hemodynamic testing [2].

Pulmonary artery pressure estimation on echocardiography has proved to be a better parameter than markers of diastolic dysfunction such as E/e' ratio, LA volume, or LV thickness. A good rule of thumb is to assume that elevated pulmonary artery systolic pressure in patients with normal ejection fraction is due to HFpEF unless proven otherwise [21].

Elevation of serological markers such as brain natriuretic peptide (BNP) and NT-pro BNP can help support diagnosis and aid in prognosis. Levels tend to be lower than in patients with HFrEF; in fact, about $30 \%$ of patients with invasive testing suggestive of elevated filling pressures $(>20 \mathrm{~mm} \mathrm{Hg}$ ) have BNP levels $<100 \mathrm{pg}$ / $\mathrm{ml}$ [22]. There is lower wall stress secondary to lower enddiastolic volumes, which is the major stimulus for BNP production by myocardial cells [23]. Obesity, seen in a large proportion of patients with HFpEF, is also associated with lower levels of NTproBNP [22].

\section{Therapeutic Options and Management}

Unlike HFrEF, no specific treatment guidelines exist, and most treatment modalities do not reduce hospitalizations and have no benefit in regard to mortality. Diuretics are key to controlling symptoms of volume overload, as seen in the Hong Kong Diastolic Heart Failure Study [24]. This study also showed no significant additional affect with irbesartan or ramipril. The combination of diuretics with inhibition of the renin-angiotensin-aldosterone system (RAAS), however, showed some benefit in LV systolic and longitudinal diastolic dysfunction with reduction in levels of NTproBNP in 1 year [24]. The ESC guidelines for management of HFpEF recommend the use of diuretics for symptom control [3]. They should be used with caution, however, given the dependence on preload in these patients.

The RAAS pathway has been the center of many trials for the treatment of HFpEF which have, to date showed mostly neutral outcomes with no benefit on mortality or number of hospitalizations [25]. The Perindopril in Elderly People with Chronic Heart Failure (PEP-CHF) study [26], which studied the effects of the drug in aged patients with HFpEF, concluded that no benefit was seen on all-cause mortality or hospitalizations. 
Perindopril did, however, improve symptoms as well as exercise capacity, with fewer hospitalizations seen in the first year. This study has been criticized for lack of power for its primary end point; thus, whether perindopril is efficacious or not remains uncertain at this point [26]. The Candesartan in Heart failure: Assessment of Reduction in Mortality and morbidity (CHARM)Preserved trial [27] studied candesartan, which was found to reduce hospitalizations but no benefit in mortality rates was seen. In the Irbesartan in Heart Failure with Preserved Ejection Fraction Study (I-PRESERVE) trial [28] with irbesartan, no benefit on mortality or hospitalizations was seen after 50 months of treatment. However, prospective analysis of the Swedish Heart Failure Registry [29] demonstrated a mortality benefit with RAAS blockade in patients with HFpEF (crude 1-year survival 86\% [95\% CI, 0.86-0.87] for treated patients vs $69 \%$ [95\% CI, 0.68-0.71] for untreated patients, HR 0.90 [95\% CI, 0.85-0.96; P = .001]). Aldosterone has also been the subject of much speculation. The Aldosterone Receptor Blockade in Diastolic Heart Failure (AldoDHF) trial [30] data showed improvement in diastolic function (95\% CI, -2.0 to -0.9 ; $\mathrm{P}<.001$ ) but did not have any positive impact on exercise capacity, symptoms, or quality of life [30]. The Treatment of Preserved Cardiac Function Heart Failure with an Aldosterone Antagonist (TOPCAT) trial [31] demonstrated no mortality benefit, although hospitalization for heart failure was reduced (206 patients [12.0\%] vs. 245 patients [14.2\%]; HR, 0.83; $95 \% \mathrm{CI}, 0.69-0.99, \mathrm{P}=0.04)]$. Based on the above data, guidelines do not recommend the use of ACE/ARB or spironolactone for HFpEF. However, they can be used where clinically appropriate for other comorbid conditions [3].

Although current recommendations do not endorse the use of beta blockers exclusively for HFpEF [3], an elevated resting heart rate has been linked to adverse outcomes [32]. Studies and registry data, however, have been inconsistent. The Study of the Effects of Nebivolol Intervention on Outcomes and Re-hospitalization in Seniors with heart failure (SENIORS) study [33] showed that the use of nebivolol improved outcomes in both HFrEF as well as HFpEF, despite having no effect on diastolic dysfunction. However, the Organized Program to Initiate Lifesaving Treatment in Hospitalized Patients with Heart Failure (OPTIMIZE-HF) registry [34] found no benefit with the use of beta blockers on hospitalizations or mortality in patients with HFpEF.

The effect of digoxin vs. placebo in patients with HFpEF was studied in the Digitalis Investigation Group (DIG) trial [35]. Although there was some reduction in hospitalization rates, there was no benefit in terms of mortality rates; in fact, mortality was increased in those with concomitant coronary artery disease [35]. The use of digoxin for the treatment of HFpEF alone is not recommended.

Statin therapy might improve mortality in HFpEF, but this effect was found to be independent of low-density-lipoprotein levels and was likely due to the protective effects of LV remodeling, LV hypertrophy, arterial distensibility, and endothelial function [36]. HFpEF patients with poor or intermediate physical activity are associated with worse outcomes than their counterparts with ideal physical activity status, as seen in the post hoc analysis of the TOPCAT trial [37]. The Exercise training in Diastolic Heart Failure (Ex-DHF) trial [38] sought to see the effects of adding a dedicated training program to guideline-recommended medical therapy in patients with HFpEF over 3 months. The investigators found that it had a favorable effect on exercise capacity and LV diastolic function [38].

\section{Future Optimization and Perspectives}

\section{Nitrates}

The use of organic nitrates such as isosorbide mononitrate is associated with poor quality of life as well as decreased exercise capacity [39]. Inorganic nitrates, on the other hand, have shown promise. One study looked into the effect of nitrate-rich beetroot juice and found that its improved exercise capacity [40]. The KNO3CKOUT-HFpEF and the Inorganic Nitrite Delivery to Improve Exercise capacity in HFpEF (INDIE-HFpEF) studies are phase II trials looking into the effects of oral and inhaled nitrites, respectively.

\section{Angiotensin-neprilysin inhibitors}

LCZ696 (sacubitril/valsartan) is another class of heartfailure medications which has generated interest as a possible therapeutic option in HFpEF. The Prospective comparison of ARNi with ARB on Management of Heart Failure with preserved ejection fraction Trial (PARAMOUNT) [41] is a phase II trial looking into the effects of LCZ696 vs. valsartan alone. A reduction of NTproBNP was observed at 12 weeks in the LCZ696 arm. Moreover, NYHA functional status improvement and reduction in atrial volumes was seen at 36 weeks [41]. These encouraging results are being investigated further by the Prospective comparison of ARNi with ARB Global Outcomes in HF with preserved ejection fraction (PARAGON-HF) trial [42], which is set to determine if any benefit in mortality rates occur.

\section{Soluble guanylyl cyclase activators and stimulators}

Direct stimulators of soluble guanylyl cyclase have achieved promising results in HFpEF. These agents mimic nitric oxide and restore downstream cGMP signaling [43]. The SOluble guanylate Cyclase stimulator in heart failure patients with PRESERVED EF (SOCRATES-PRESERVED) trial [44] reported no effect of vericiguat on NT-proBNP levels when compared to placebo. The drug was well tolerated, however, and improved quality of life.

\section{Cytokine inhibition}

Chronic systemic inflammation brings about higher levels of interleukin1, which has been implicated in promoting myocardial stiffness in HFpEF. In one small study [45], interleukin-1 blockade with anakinra was shown to decrease C-reactive protein levels, leading to improved peak oxygen consumption.

\section{Drugs traditionally used to treat diabetes}

The Pioglitazone Influence on triglyceride Accumulation in the Myocardium in Diabetes (PIRAMID) study [46] showed 
improvement of diastolic function as well as myocardial glucose uptake with the use of pioglitazone in patients with type 2 diabetes mellitus. Glucagon-like peptide-1 (GLP-1) receptors are found in the heart, and their stimulation has been found to activate cardiac glycolysis [47]. These receptors are stimulated by both GLP-1 analogs as well as dipeptidyl peptidase inhibitor-4 inhibitors. GLP-1 analogs are potential targets for use in HFpEF; in one study in an animal model, GLP-1 showed a favorable effect on cardiomyocyte metabolism and diastolic function, independent of the level of insulin [48]. In diabetic patients at high cardiovascular risk, semaglutide demonstrated a significant improvement regarding mortality [49]. Sitagliptin, an inhibitor of dipeptidyl peptidase inhibitor-4, was associated with an improvement in myocardial glucose uptake in nondiabetic patients with nonischemic cardiomyopathy [50].

The BI 10773 (Empagliflozin) Cardiovascular Outcome Event Trial in Type 2 Diabetes Mellitus Patients (EMPA-REG OUTCOME trial) [51] compared the use of empagliflozin, a sodium-glucose co-transporter 2 inhibitor, vs. placebo. The trial data showed that patients in the treatment arm had lower all-cause mortality and cardiovascular mortality as well as fewer hospitalizations for heart failure; they also had a significant decrease in systolic blood pressures [51].

\section{Device therapy}

\section{CORolla ${ }^{\circledR}$}

The CORolla ${ }^{\circledR}$ (CorAssist Cardiovascular Ltd., Haifa, Israel) is an elastic device designed to help with ventricular filling during diastole. It is implanted into the beating heart via a minimally invasive transapical or transcutaneous approach. It is yet to be determined if this device is effective; however, it looks promising and was recently implanted for the first time in a 72-year-old male with HFpEF [52].

\section{Atrial shunt devices}

Reduction of LA pressure via a small artificial left-to-right shunt has been hypothesized as a treatment modality for HFpEF. In a study of 11 patients with HFpEF, an interatrial device was used to create a small interatrial shunt via a catheter-based technique. Mean filling pressures decreased and mean NYHA functional status improved without the development of pulmonary hypertension at 30 days [53]. The Reduce Elevated Left Atrial Pressure in Patients with Heart Failure (REDUCE LAP-HF) randomized trial I [54] was a multicenter, single-arm, phase- 1 trial that evaluated the implantation of the interatrial shunt device. More than $50 \%$ of the 68 patients in the study had a reduction in wedge pressure at rest or exertion [54].

Cardiac resynchronization therapy and cardiac contractility modulation

Mechanical dyssynchrony has been demonstrated in patients with HFpEF (not dissimilar to patients with HFrEF and a narrow QRS duration), which has raised the possibility of the use of cardiac resynchronization therapy in HFpEF [55]. Cardiac contractility modulation (CCM) delivers a nonexcitatory electric current into the cardiac septum during the absolute refractory period to improve cardiac contraction as well as remodeling. Although more extensively studied in HFrEF, subgroup analysis reveals better outcomes in those with higher baseline ejection fractions [56]. CCM has shown promise as a treatment for heart failure, and its use has resulted in an improvement in NYHA functional status and exercise capacity as well as diastolic function [57].

\section{Summary and Conclusion}

HFpEF accounts for $50 \%$ of hospitalizations for heart failure. It was traditionally described as diastolic dysfunction and was thought to be primarily secondary to LV hypertrophy in the setting of chronic hypertension. Newer studies have suggested that multiple other factors play a role; hence there has been a shift in terminology, from diastolic dysfunction to HFpEF. Therapy directed at HFrEF has not shown any lowering of mortality rates in patients with HFpEF. Currently, treatment is directed at treating symptoms as well as comorbidities such as hypertension, diabetes, and obesity. As we come to better understand the pathophysiology of HFpEF, newer therapeutic options have emerged as a subject of ongoing clinical trials. However, there is still much to be learned, and more research is needed in this area.

\section{References}

1. Gladden JD, Linke WA, Redfield MM (2014) Heart failure with preserved ejection fraction. Pflügers Arch 466(6): 1037-1053.

2. Borlaug BA, Paulus WJ (2011) Heart failure with preserved ejection: pathophysiology, diagnosis, and treatment. Eur Heart J 32(6): 670-679.

3. Ponikowski P, Voors AA, Anker SD, Bueno H, Cleland JGF, et al. (2016) 2016 ESC Scientific Document Group. 2016 ESC Guidelines for the diagnosis and treatment of acute and chronic heart failure: the task force for the diagnosis and treatment of acute and chronic heart failure of the European Society of Cardiology (ESC). Developed with the special contribution of the Heart Failure Association (HFA) of the ESC. Eur Heart J 37(27): 2129-2200.

4. Lourenço AP, Leite-Moreira AF, Balligand JL, Bauersachs J, Dawson D, et al. (2018) An integrative translational approach to study heart failure with preserved ejection fraction: a position paper from the Working Group on Myocardial Function of the European Society of Cardiology. Eur J Heart Fail 20(2): 216-227.

5. Tschöpe C, Birner C, Böhm M, Bruder O, Frantz S, et al. (2018) Heart failure with preserved ejection fraction: current management and future strategies. Expert opinion on the behalf of the Nucleus of the "Heart Failure Working Group" of the German Society of Cardiology (DKG). Clin Res Cardiol 107(1): 1-19.

6. Loffredo FS, Nikolova AP, Pancoast JR, Lee RT (2014) Heart failure with preserved ejection fraction: molecular pathways of the aging myocardium. Circ Res 115(1): 97-107.

7. Upadhya B, Pisani B, Kitzman DW (2017) Evolution of a geriatric syndrome: pathophysiology and treatment of heart failure with preserved ejection fraction. J Am Geriatr Soc 65(11): 2431-2440.

8. Tsujimoto T, Kajio H (2017) Abdominal obesity is associated with an increased risk of all-cause mortality in patients with HFpEF. J Am Coll Cardiol 70(22): 2739-2749.

9. Kristensen SL, Mogensen UM, Jhund PS (2017) Clinical and echocardiographic characteristics and cardiovascular outcomes according to diabetes status in patients with heart failure and preserved 
ejection fraction: a report from the I-Preserve Trial (irbesartan in heart failure with preserved ejection fraction). Circulation 135(8): 724-735.

10. Haykowsky MJ, Tomczak CR, Scott JM, Paterson DI, Kitzman DW (2015) Determinants of exercise intolerance in patients with heart failure and reduced or preserved ejection fraction. J Appl Physiol 119(6): 739-744.

11. Kitzman DW, Guazzi M (2016) Impaired alveolar capillary membrane diffusion: a recently recognized contributor to exertional dyspnea in heart failure with preserved ejection fraction. JACC Heart Fail 4(6): 499-501.

12. Dunlay SM, Weston SA, Redfield MM, Killian JM, Roger VL (2008) Anemia and heart failure: a community study. Am J Med 121(8): 726732.

13. Anker SD, Comin Colet J, Filippatos G, Willenheimer R, Dickstein K, et al. (2009) FAIR-HF Trial Investigators. Ferric carboxymaltose in patients with heart failure and iron deficiency. New Engl J Med 361(25): 2436 2448.

14. Maurer MS, Teruya S, Chakraborty B, Helmke S, Mancini D (2013) Treating anemia in older adults with heart failure with a preserved ejection fraction with epoetin alfa: single-blind randomized clinical trial of safety and efficacy. Circ Heart Fail; 6(2): 254-263.

15. Zile MR, Little WC (2015) Heart failure with a preserved ejection fraction. In: Mann DL, Zipes DP, Libby PM, Bonow RO, (eds.), Braunwald's Heart Disease: A Textbook of Cardiovascular Medicine. 10th ed. Philadelphia, PA: Elsevier/Saunders pp. 557-574.

16. Kawaguchi M, Hay I, Fetics B, Kass DA (2003) Combined ventricular systolic and arterial stiffening in patients with heart failure and preserved ejection fraction: implications for systolic and diastolic reserve limitations. Circulation 107(5): 714-720.

17. Yu CM, Lin H, Yang H, Kong SL, Zhang Q et al. (2002) Progression of systolic abnormalities in patients with "isolated" diastolic heart failure and diastolic dysfunction. Circulation 105(10): 1195-1201.

18. Borlaug BA, Nishimura RA, Sorajja P, Lam CS, Redfield MM (2010) Exercise hemodynamics enhance diagnosis of early heart failure with preserved ejection fraction. Circ Heart Fail 3(5): 588-595.

19. Dhakal BP, Malhotra R, Murphy RM (2015) Mechanisms of exercise intolerance in heart failure with preserved ejection fraction: the role of abnormal peripheral oxygen extraction. Circ Heart Fail 8(2): 286-294.

20. Haykowsky MJ, Brubaker PH, Stewart KP, Morgan TM, Eggebeen J, et al. (2012) Effect of endurance training on the determinants of peak exercise oxygen consumption in elderly patients with stable compensated heart failure and preserved ejection fraction. J Am Coll Cardiol 60(2): 120-128.

21. Lam CS, Roger VL, Rodeheffer RJ, Borlaug BA, Enders FT, et al. (2009) Pulmonary hypertension in heart failure with preserved ejection fraction: a community-based study. J Am Coll Cardiol 53(13): 11191126.

22. Anjan VY, Loftus TM, Burke MA, Akhter N, Fonarow GC, et al. (2012) Prevalence, clinical phenotype, and outcomes associated with normal B-type natriuretic peptide levels in heart failure with preserved ejection fraction. Am J Cardiol 110(6): 870-876.

23. Iwanaga Y, Nishi I, Furuichi S (2005) B-type natriuretic peptide strongly reflects diastolic wall stress in patients with chronic heart failure: comparison between systolic and diastolic heart failure. J Am Coll Cardiol 2006 47(4): 742-748.

24. Yip GW, Wang M, Wang T, Chan S, Fung JW, et al. (2008) The Hong Kong diastolic heart failure study: a randomised controlled trial of diuretics, irbesartan and ramipril on quality of life, exercise capacity, left ventricular global and regional function in heart failure with a normal ejection fraction. Heart 94(5): 573-580.
25. Shah RV, Desai AS, Givertz MM (2010) The effect of renin-angiotensin system inhibitors on mortality and heart failure hospitalization in patients with heart failure and preserved ejection fraction: a systematic review and meta-analysis. J Card Fail 16(3): 260-267.

26. Cleland JGF, Tendera M, Adamus J, Freemantle N, Polonski L, et al. (2006) PEP-CHF Investigators. The perindopril in elderly people with chronic heart failure (PEP-CHF) study. Eur Heart J 27(19): 2338-2345.

27. Yusuf S, Pfeffer MA, Swedberg K, Granger CB, Held P, et al. (2003) Effects of candesartan in patients with chronic heart failure and preserved left-ventricular ejection fraction: the CHARM-Preserved Trial. Lancet 362(9386): 777-781.

28. Massie BM, Carson PE, McMurray JJ, Komajda M, McKelvie R, et al. (2008) Irbesartan in patients with heart failure and preserved ejection fraction. New Engl J Med 359(23): 2456-2467.

29. Lund LH, Benson L, Dahlström U, Edner M (2012) Association between use of renin-angiotensin system antagonists and mortality in patients with heart failure and preserved ejection fraction. JAMA 308(20): 2108-2117.

30. Edelmann F, Wachter R, Schmidt AG, Kraigher-Krainer E, Colantonio C, et al. (2013) Aldo-DHF Investigators. Effect of spironolactone on diastolic function and exercise capacity in patients with heart failure with preserved ejection fraction: the Aldo-DHF randomized controlled trial. JAMA 309(8): 781-791.

31. Pitt B, Pfeffer MA, Assmann SF, Boineau R, Anand IS, et al. (2014) Spironolactone for heart failure with preserved ejection fraction. New Engl J Med 370(15): 1383-1392.

32. O’Neal WT, Sandesara PB, Samman-Tahhan A, Kelli HM, Hammadah M, et al. (2017) Heart rate and the risk of adverse outcomes in patients with heart failure with preserved ejection fraction. Eur J Prev Cardiol 24(11): 1212-1219.

33. Flather MD, Shibata MC, Coats AJ, Van Veldhuisen DJ, Parkhomenko A, et al. (2005) SENIORS Investigators. Randomized trial to determine the effect of nebivolol on mortality and cardiovascular hospital admission in elderly patients with heart failure (SENIORS). Eur Heart J 26(3): 215-225.

34. Hernandez AF, Hammill BG, O'Connor CM, Schulman KA, Curtis LH, et al. (2009) Clinical effectiveness of beta-blockers in heart failure: findings from the OPTIMIZE-HF (Organized Program to Initiate Lifesaving Treatment in Hospitalized Patients with Heart Failure) Registry. J Am Coll Cardiol 53(2): 184-192.

35. Ahmed A, Rich MW, Fleg JL, Zile MR, Young JB, et al. (2006) Effects of digoxin on morbidity and mortality in diastolic heart failure: the ancillary digitalis investigation group trial. Circulation 114(5): 397403.

36. Fukuta H, Goto T, Wakami K, Ohte N (2016) The effect of statins on mortality in heart failure with preserved ejection fraction: a metaanalysis of propensity score analyses. Int J Cardiol 214: 301-306.

37. Hegde SM, Claggett B, Shah AM, Lewis EF, Anand I, et al. (2017) Physical activity and prognosis in the TOPCAT Trial (treatment of preserved cardiac function heart failure with an aldosterone antagonist). Circulation 136(11): 982-992.

38. Edelmann F, Gelbrich G, Düngen HD, Fröhling S, Wachter R, et al. (2011) Exercise training improves exercise capacity and diastolic function in patients with heart failure with preserved ejection fraction: results of the Ex-DHF (Exercise training in Diastolic Heart Failure) pilot study. J Am Coll Cardiol 58(17): 1780-1791.

39. Redfield MM, Anstrom KJ, Levine JA, Koepp GA, Borlaug BA, et al. (2015) Heart Failure Clinical Research Network. Isosorbide mononitrate in heart failure with preserved ejection fraction. New Engl J Med 373(24): 2314-2324. 
40. Zamani P, Rawat D, Shiva-Kumar P, Geraci S, Bhuva R, et al. (2015) Effect of inorganic nitrate on exercise capacity in heart failure with preserved ejection fraction. Circulation 131(4): 371-380.

41. Solomon SD, Zile M, Pieske B, Voors A, Shah A, et al. (2012) Prospective comparison of ARNI with ARB on Management of heart failUre with preserved ejection fracTion (PARAMOUNT) Investigators. The angiotensin receptor neprilysin inhibitor LCZ696 in heart failure with preserved ejection fraction: a phase 2 double-blind randomised controlled trial. Lancet 380 (9851):1387-1395.

42. Solomon SD, Rizkala AR, Gong J, Wang W, Anand IS, et al. (2017) Angiotensin receptor neprilysin inhibition in heart failure with preserved ejection fraction: rationale and design of the PARAGON-HF trial. JACC Heart Fail 5(7): 471-482.

43. Gheorghiade M, Greene SJ, Butler J, Filippatos G, Lam CS, et al. (2015) Effect of vericiguat, a soluble guanylate cyclase stimulator, on natriuretic peptide levels in patients with worsening chronic heart failure and reduced ejection fraction: the SOCRATES-REDUCED Randomized Trial. JAMA 314(21): 2251-2262.

44. Pieske B, Maggioni AP, Lam CSP, Pieske-Kraigher E, Filippatos G, et al (2017) Vericiguat in patients with worsening chronic heart failure and preserved ejection fraction: results of the SOluble guanylate Cyclase stimulatoR in heArT failurE patientS with PRESERVED EF (SOCRATESPRESERVED) study. Eur Heart J 38(15): 1119-1127.

45. Van Tassell BW, Arena R, Biondi-Zoccai G, Canada JM, Oddi C, et al (2014) Effects of interleukin-1 blockade with anakinra on aerobic exercise capacity in patients with heart failure and preserved ejection fraction (from the D-HART pilot study). Am J Cardiol 113(2): 321-327.

46. Van der Meer RW, Rijzewijk LJ, de Jong HW, Lamb HJ, Lubberink $\mathrm{M}$, et al. (2009) Pioglitazone improves cardiac function and alters myocardial substrate metabolism without affecting cardiac triglyceride accumulation and high-energy phosphate metabolism in patients with well-controlled type 2 diabetes mellitus. Circulation 119(15): 20692077.

47. Inzucchi SE, McGuire DK (2008) New drugs for the treatment of diabetes: Part II: incretin-based therapy and beyond. Circulation 117(4): 574-584.
48. Nguyen TD, Shingu Y, Amorim PA, Schenkl C, Schwarzer M, et al. (2018) GLP-1 improves diastolic function and survival in heart failure with preserved ejection fraction. J Cardiovasc Transl Res 11(3): 259-267.

49. Marso SP, Bain SC, Consoli A, Eliaschewitz FG, Jódar E, et al. (2016) SUSTAIN-6 Investigators. Semaglutide and cardiovascular outcomes in patients with type 2 diabetes. New Engl J Med 357(19): 1834-1844.

50. Witteles RM, Keu KV, Quon A, Tavana H, Fowler MB (2012) Dipeptidyl peptidase 4 inhibition increases myocardial glucose uptake in nonischemic cardiomyopathy. J Card Fail 18(10): 804-809.

51.Zinman B, Wanner C, Lachin JM, Fitchett D, Bluhmki E, et al. (2015) Empagliflozin, cardiovascular outcomes, and mortality in type 2 diabetes. New Engl J Med 373(22): 2117-2128.

52. CorAssist Cadiovascular Ltd (2018) CORolla ${ }^{\mathrm{TM}}$ TAA for Heart Failure and Preserved Ejection Fraction and Diastolic Dysfunction.

53. Søndergaard L, Reddy V, Kaye D, Malek F, Walton A, et al. (2014) Transcatheter treatment of heart failure with preserved or mildly reduced ejection fraction using a novel interatrial implant to lower left atrial pressure. Eur J Heart Fail 16(7): 796-801.

54. Hasenfu G, Hayward C, Burkhoff D, Silvestry FE, McKenzie S, et al. (2016) REDUCE LAP-HF study investigators. A transcatheter intracardiac shunt device for heart failure with preserved ejection fraction (REDUCE LAP-HF): a multicentre, open-label, single-arm, phase 1 trial. Lancet 387(10025): 1298-1304.

55. Menet A, Greffe L, Ennezat PV, Delelis F, Guyomar Y, et al. (2014) Is mechanical dyssynchrony a therapeutic target in heart failure with preserved ejection fraction? Am Heart J 168(6): 909.e1-916.e1.

56. Borggrefe M, Burkhoff D (2012) Clinical effects of cardiac contractility modulation (CCM) as a treatment for chronic heart failure. Eur J Heart Fail 14(7): 703-712.

57. Abi-Samra F, Gutterman D (2016) Cardiac contractility modulation: a novel approach for the treatment of heart failure. Heart Fail Rev 21(6): 645-660.

Your next submission with Juniper Publishers
will reach you the below assets
- Quality Editorial service
- Swift Peer Review
- Reprints availability
- E-prints Service
- Manuscript Podcast for convenient understanding
- Global attainment for your research
- Manuscript accessibility in different formats
( Pdf, E-pub, Full Text, Audio)
- Unceasing customer service
Track the below URL for one-step submission
https://juniperpublishers.com/online-submission.php

